\title{
Application of Fault Location Based on Detecting Cycles Covering Technology in Optical Burst Switching Network
}

\author{
Bao Song-jian \\ College of Electronic and Electrical Engineering, ChongQing University of Arts and \\ Sciences, Chongqing 402160, China \\ email:bsj126@126.com
}

Keywords: Detecting circle covering technology;fault location mechanism;alarm information; effective positioning

\begin{abstract}
The identify and locate of optical network faults are very important, some existing fault location algorithm is lack of applicability and low locating rate in the interference alarm information. According to this, this paper proposed the corresponding fault location mechanism on the basis of the shortest length $\mathrm{m}$ circle algorithm. The fault location mechanism is divided into two steps, the first step: collect alarm information by fault triggered, and compose corresponding binary alarm correlation matrix; The second step: adopt pre-computation way, locate faults through the alarm matrix, which can in the case of both avoiding leakage alarm and false alarm, solve the problem of multiple faults location, find the possible fault equipment sets, finally realize the effective positioning.
\end{abstract}

\section{Introduction}

The identify and locate of optical network faults are very important, some existing fault location algorithms are mainly rate algorithm, data mining algorithm and the correlation algorithm in graph theory, but these algorithms for optical burst switching (OBS) network are lack of applicability.

This paper proposed the corresponding fault location mechanism on the basis of the shortest length $\mathrm{m}$ circle algorithm. The fault location mechanism is divided into two steps, the first step: collect alarm information by fault triggered, and compose corresponding binary alarm correlation matrix; The second step: adopt pre-computation way, locate faults through the alarm matrix, which can in the case of both avoiding leakage alarm and false alarm, solve the problem of multiple faults location, find the possible fault equipment sets, finally realize the effective positioning.

\section{Fault Detection and Localization Algorithm in OBS Networks}

In OBS networks, both single fault and multiple faults can be alarm detect and alert, in order to reduce the complexity of the fault management and cost, try to achieve with the least amount of monitoring equipment to monitor the OBS network all elements.

Interference alarms (false alarm and leakage alarm) appear, which make the difficulty of fault location, the fault location scheme mainly can be divided into two steps: the first step: collect and detect all kinds of alarm information of OBS network elements. The second step: use the below mentioned fault location algorithm, and finally get the set of OBS network fault equipment, the specific process as shown in Fig.1.

After receiving the alarm from monitoring equipment, whatever the type of alarm, fault location algorithm will run, and the just received alarm information compared with the calculated beforehand alarm matrix, during the process of this comparison, it may be produce sets of the OBS network fault equipment, and then process and analyze emitting and receiving signal, determine the accurate fault equipment from the sets. 


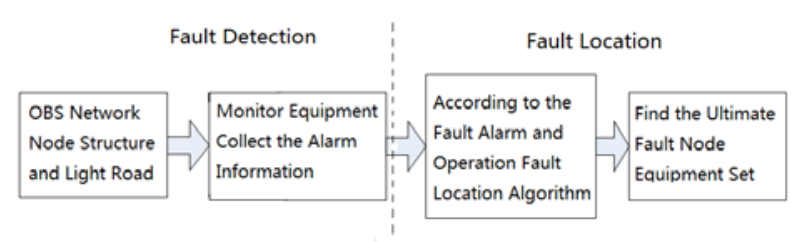

Fig.1 OBS network fault location scheme

\section{OBS Network Node Fault Location Algorithm.}

The survivability of fault location just puts the fault location to the resource ${ }^{[1]}$. Resource is relative to the business. Here is a kind of fault location mechanism, in the mechanism $C$ represents a collection of all the OBS network devices, $M$ represents all the monitor set, Domain $(v)$ represents OBS optical network device $v$ a warning field (when $v$ occurs fault, which causes alarm monitoring equipment collection). We also define the bell ring surveillance equipment set which is represented by the ${ }^{M_{\mathrm{R}}}, M_{\mathrm{R}} \subseteq M$; by the same token, the ${ }^{M_{\mathrm{s}}}$ is defined the no ring surveillance equipment set, $M_{\mathrm{s}}=M / M_{R}$.

The $C^{\prime}$ is a subset of the set $C$. we also define $M_{A}\left(C^{\prime}\right)$ as in the case of no false alarm and leakage alarm. When all the equipment failure occurs in the $C^{\prime}$, the monitor will send out a normal alarm. So when the alarm disturbance under the condition, $M_{A}\left(C^{\prime}\right) \neq M_{R}$. The false alarms can be expressed as $M_{F}\left(C^{\prime}\right)=M_{R} / M_{A}\left(C^{\prime}\right)$. Similarly, leakage alarm can be expressed as $M_{M}\left(C^{\prime}\right)=M_{S} \cap M_{A}\left(C^{\prime}\right)$. The goal of multiple fault location is all the alarm in the covered alarm bell ring matrix (i.e., alarms in the alarm matrix is 1.) rather than not alarm bell ring (i.e., alarms in the alarm matrix is 0 .), try to make the final fault equipment set is the minimum and achieve the purpose of accurate positioning. So we want to find a subset $C^{\prime}$ of $C$. The number of the fault equipment sets $\left|C^{\prime}\right|$ is the minimum as far as possible. When there is no false alarm, the above situation meets formula 1 :

$$
M_{F}\left(C^{\prime}\right)=\phi
$$

The absence of leakage alarm content formula 2:

$$
M_{n}\left(C^{\prime}\right)=\phi
$$

In positioning algorithm, it is necessary to consider the following four kind of situations:

1) No false alarm and leakage alarm fault location.Actually, more ideally fault location problem is equivalent to a set cover problem. Set cover problem is a complete NP problem ${ }^{[2]}$. In order to solve this problem, this paper references Sets Cover Adaptive Greed Optimization Algorithm for the multiple fault locating. The algorithm repeatedly selects failure equipment which is much more ring the alarm in the alarm matrix.

For any one of OBS network topology, the first, a warning matrix is got through the detecting cycle algorithm, and then run to its fault location algorithm which satisfy the conditions $\operatorname{Domain}(c) \subseteq M_{R}$. We received the warning coding during localization algorithm run matrix of each network device (nodes) positioning compared based on its condition, namely meet formula 3 and 4.

$$
\begin{aligned}
& M_{R}=M_{R} / \operatorname{Domain}(c) \\
& \operatorname{Domain}\left(c_{i}\right)=\operatorname{Domain}\left(c_{i}\right) / \operatorname{Domain}(c)
\end{aligned}
$$

2) A false alarm but the absence of leakage alarm. OBS network effectively supports the upper agreement or sudden business of high-level user generated through time domain share and statistical multiplexing optical wavelength channel. So with or without the channel optical signals, which are random, Loss-of-light doesn't mean that there must be a fault event occurs. This makes by monitoring the signal of the presence of light to determine whether to produce the alarm fault monitoring which will produce too many false alarms ${ }^{[3]}$. So that the fault location algorithm can't locate the fault, or because the warning threshold value set in surveillance equipment is low, it can 
also cause the situation. So when I received the alarm bell ring set $M_{\mathrm{R}}$, in which there may be some false alarm, we need to identify a set of network equipment $F_{D} \subseteq C$, which has the least number of false alarm (minimum) and satisfy the formula 2. The algorithm is as follows:

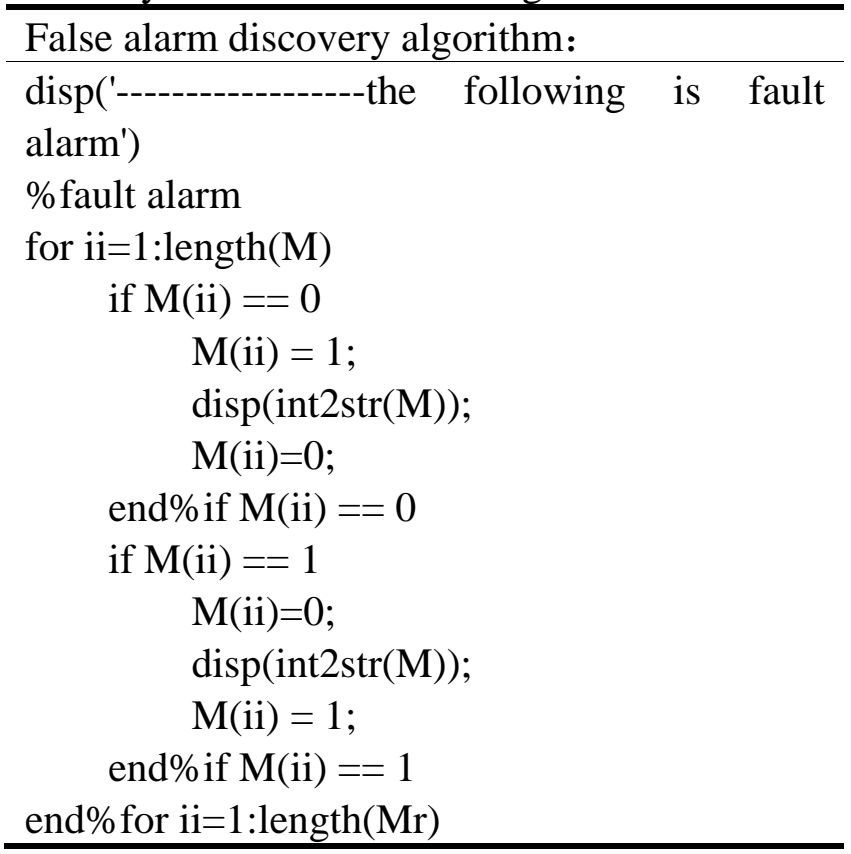

3 ) A leakage alarm but the absence of false alarms. This is because the warning threshold value in monitoring equipment is set up high. We need to identify a network equipment set $F_{D} \subseteq C$. It has the least number of leakage alarm $\left(\left|M_{M}\left(F_{D}\right)\right|\right.$ is minimum) and satisfy the formula 1 . Due to leakage alarm problem is a NP problem completely, so the relative to false alarms can be much more difficult to correct ${ }^{[4]}$, and it should set the monitor alarm threshold to make the number of leakage alarm at the least, in order to reduce the interference in the process of fault location. The algorithm is as follows:

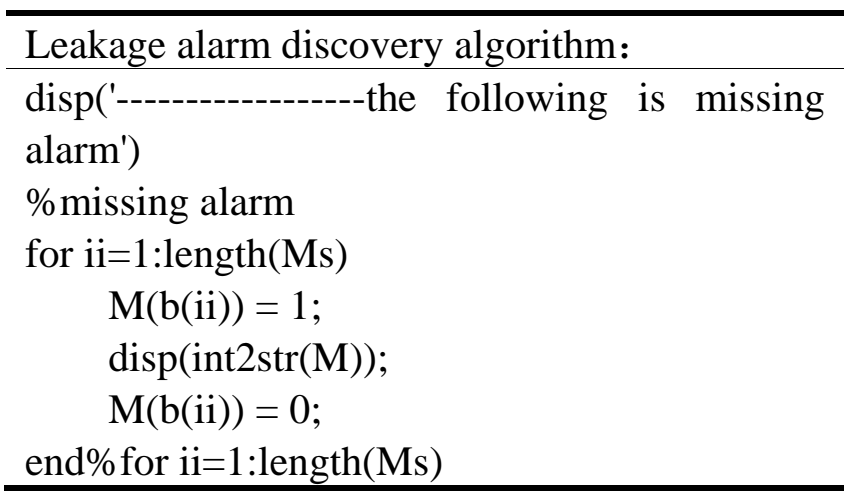

4) A false alarm and a leakage alarm. In OBS network, the network operation condition is very complex. It is difficult to determine only a leakage alarm or only a false alarm occurs. So we put forward a algorithm as far as possible to consider both occur together. The algorithm is as follows:

Leakage alarm and false alarm discovery

algorithm:

disp('--------------the following is missing
alarm and fault alarm')
\%missing alarm and fault alarm
for ii=1:length(Ms)
for $\mathrm{jj}=1:$ length(Mr)
$\mathrm{M}(\mathrm{b}(\mathrm{ii}))=1$;
$\mathrm{M}(\mathrm{a}(\mathrm{jj}))=0$;




\begin{tabular}{c}
\hline $\operatorname{disp}(\operatorname{int} 2 \mathrm{str}(\mathrm{M})) ;$ \\
$\mathrm{M}(\mathrm{a}(\mathrm{jj}))=1 ;$ \\
end $\%$ for $\mathrm{jj}=1$ :length(Mr) \\
$\mathrm{M}(\mathrm{b}(\mathrm{ii}))=0 ;$ \\
end\%for ii=1:length(Ms)
\end{tabular}

Fault location algorithm based on detecting cycle is considered the four kind of situations: (1) No false alarm and leakage alarm.(2) A false alarm but no leakage alarm.(3) A leakage alarm and no false alarm.(4) A false alarm and a leakage alarm. For example, the algorithm is explained by table 1.

For any one OBS network topology, it makes sure to find all the shortest length $\mathrm{m}^{2}$ cycle, then the alarm matrix as shown in table 2. If an alarm set $\left\{\begin{array}{llllll}1 & 1 & 1 & 0 & 0\end{array}\right\}$ is received, so that $M_{3}, M_{6}$ and $M_{7}$ will trigger the alarm, but, $M_{1}$ and $M_{13}$ will not trigger the alarm. These are normal alarm for the situation (1). Now, the alarm of the network node 4 may be expressed as Domain(ND4) $\subseteq M_{R}$. Similarly, for the network nodes 6, 10 and 12, Domain(ND6) $\subseteq M_{R}, \operatorname{Domain}(N D 10) \subseteq M_{R}$ and $\operatorname{Domain}(N D 12) \subseteq M_{R}$. According to the fault location algorithm, the got ND4, ND6, ND10, ND12 \} belongs to faulty component FC. There is a false alarm for the situation (2). The found alarm sets will be more than three $\left\{\left(\begin{array}{lllll}1 & 1 & 0 & 0 & 0\end{array}\right),\left(\begin{array}{lllll}1 & 0 & 1 & 0 & 0\end{array}\right),\left(\begin{array}{lllll}0 & 1 & 1 & 0 & 0\end{array}\right)\right\}$. For the situation (3), the alarm set will

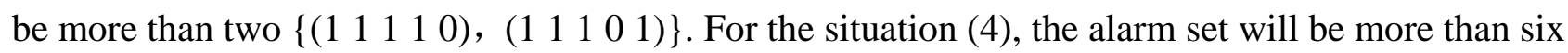

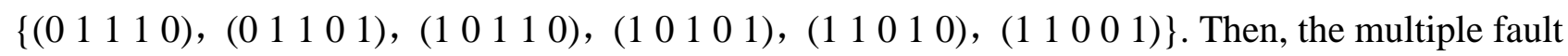
location algorithm is run for locating respectively.

Tab.1 Alarm correlation matrix

\begin{tabular}{|l|l|l|l|l|l|}
\hline & $M_{7}$ & $M_{3}$ & $M_{6}$ & $M_{13}$ & $M_{1}$ \\
\hline ND4 & 1 & 0 & 0 & 0 & 0 \\
\hline ND5 & 1 & 0 & 1 & 0 & 1 \\
\hline ND6 & 0 & 1 & 0 & 0 & 0 \\
\hline ND7 & 0 & 1 & 0 & 1 & 0 \\
\hline ND8 & 0 & 0 & 0 & 1 & 0 \\
\hline ND10 & 1 & 0 & 1 & 0 & 0 \\
\hline ND12 & 0 & 0 & 1 & 0 & 0 \\
\hline
\end{tabular}

\section{Simulation Performance Comparison}

C.Mas has made the study for the fault detection and localization of WDM optical network, and the simulation performance proves that the binary tree model is a rapid and effective fault location algorithm ${ }^{[5]}$. But with the increase of network node and fault count, the binary tree branches number and the number of the root node will be rapid growth. So it needs large storage space, and finally to be improved the accuracy of fault location. The algorithm proposed in this paper is a comprehensive consideration based on the above features. When an alarm code is received, considering the situations of all the leakage alarm and false alarm, we find each case of OBS network fault equipment number, then calculate whose the average; Similarly, the average number of fault equipment of the traditional binary tree algorithm is found. The two localization algorithm is compared, the performance comparison as shown in Fig. 2 and Fig.3:

By analyzing the operation process of C. Mas binary tree fault location algorithm, the following conclusions are,

1) the building process of network depend on the model binary tree is complex. The amount and type of network alarm equipment cannot be too little, or no enough warning information is correlate, and accurate fault location doesn't realize. If the input of the alarm information is 0001010000000 , this algorithm obtain the result of a single fault location which is not the only and led to that the fault location accuracy is reduced in the situation of the multiple failure and leakage (mistakenly). 
2) In the alarm equipment concentration, if there is only one warning alarm, when leakage alarm occurs, the algorithm will not be able to locate the network fault equipment.

3) This kind of method based on channel coding, the alarm equipment set of the last a device of each channel is null. If the equipment occurs fault, which is not located. And the fault location algorithm based on detecting cycle considers leakage (mistakenly) alarm. At the same time, because the reference set covering the adaptive optimization algorithm is greedy, so in the above aspects, the algorithm has very big improvement. It just need to find the alarm field contains up to ring the alarm failure equipment.

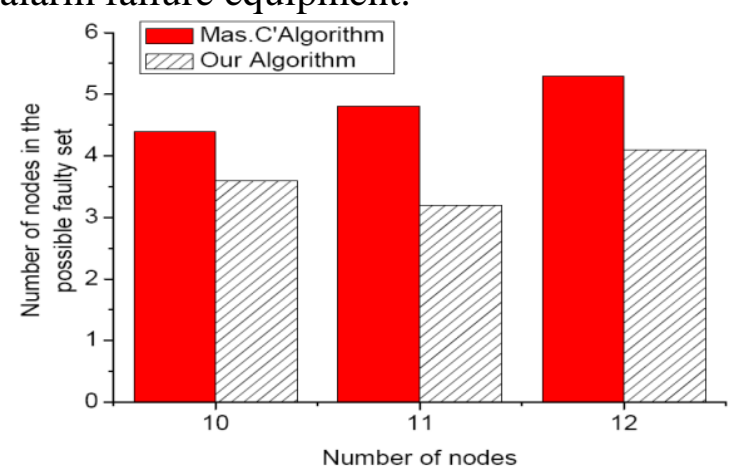

Fig.2. Comparison under the single fault location accuracy

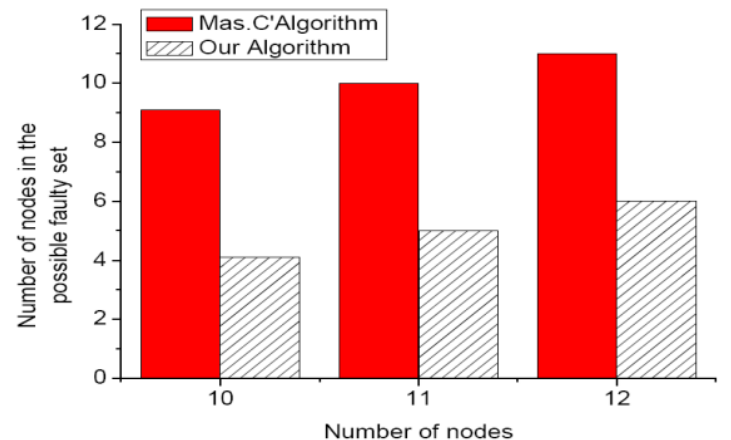

Fig.3. Comparison under the two faults location accuracy

From figure 2 and figure 3, in the same the condition of considering leakage alarm and false alarm, fault location algorithm based on detecting cycle either in single fault or multiple fault, finds that the fault equipment number is smaller eventually. It makes the fault management can reduce the fault search range and achieve accurate positioning.

\section{Conclusions}

According to the characteristics of OBS network topology, in this paper, a fault location scheme based on detecting cycle cover is proposed. This scheme is aimed at OBS network link and node dual localization and makes more accurate fault location. For a network topology, firstly using $\mathrm{m}^{2}$ cycle algorithm builds fault monitor and nodes in network topology and link correlation model. then the fault location respectively is run under considering the common interference such as leakage alarm and false alarm. By the simulation results, it can be seen in the same consider interference condition of the alarm, the proposed fault location algorithm based on detecting cycle fault location relative to the traditional binary tree algorithm has more precise positioning scope, which can achieve OBS network fault location quickly and efficiently.

\section{References}

[1] 3Ruyan Wang, Jiaofa Chang, Keping Long. A Fault Detection and Location Mechanism for Optical Burst Switching Networks [J]. Journal of Optoelectronics. Laser. 2006, 17(12): 1477-1481.

[2] Hochbaum, D., et al. Approximation Algorithms for NP-Hard Problems[J]. PWS Publishing Company, Boston, MA (1997).

[3] Carr, R.D., Doddi, S., Konjevod, G., Marathe, M.. On the red-blue set cover problem[C]. //Proceedings of Symposium on Discrete Algorithms. 2000.

[4] P. Nayek, S. Pal, B. Choudhury, A. Mukherjee, D. Saha and M. Nasipuri. Optimal Monitor Placement Scheme for Single Fault Detection in Optical Network[C]//Transparent Optical NetworksI CTON .2005, Barcelona, Spain, July 3-7, 2005.

[5] Camen Mas, Patrick Thiran. An efficient algorithm for locating soft and hard failures in WDM networks[J]. IEEE Journal on Selected Areas in Communications. 2000,18(10):1900-1911. 